\title{
MULTIDIMENSIONAL HAUSDORFF OPERATORS ON THE REAL HARDY SPACE
}

\author{
A. K. LERNER and E. LIFLYAND ${ }^{\unlhd}$
}

(Received 27 October 2005; revised 25 April 2006)

Communicated by G. Willis

\begin{abstract}
For a wide family of multivariate Hausdorff operators, the boundedness of an operator from this family is proved on the real Hardy space. By this we extend and strengthen previous results due to Andersen and Móricz.
\end{abstract}

2000 Mathematics subject classification: primary 47B38, 42B10; secondary 46E30. Keywords and phrases: Hausdorff operator, Fourier transform, real Hardy space.

\section{Introduction}

Hausdorff means, the Cesàro means among them, were known long ago; a chapter is devoted to them and their application to the summation of number series in the celebrated book 'Divergent series' [12] by Hardy. One can also find a brief survey on the Hausdorff summability and its application to the moment problem in [20, Chapter III, Section 7]. Cesàro means for power series from the Hardy space $H^{1}$ were considered by Siskakis. The idea was to substitute the coefficients $a_{k}$ in the expansion $f(z)=\sum_{k=0}^{\infty} a_{k} z^{k}$, with $f \in H^{1}$, for their Cesàro means $k^{-1} \sum_{p=0}^{k-1} a_{p}$ in order to improve the behavior of the series on the unit circle; for an elegant proof of the boundedness of the corresponding operator in $H^{1}$, see [17].

The Fourier transform setting of the correspondent problem was considered in [9]. In fact, general Hausdorff means of a Fourier-Stieltjes transform were introduced even earlier, in [8], but only in $L^{1}$. In the real Hardy space on $\mathbb{R}$, for the Hausdorff operator

(C) 2007 Australian Mathematical Society 1446-7887/07 $\$ A 2.00+0.00$ 
defined by means of $\varphi \in L^{1}(\mathbb{R})$, as

$$
(\mathcal{H} f)(x)=\left(\mathcal{H}_{\varphi} f\right)(x)=\int_{\mathbb{R}} \frac{\varphi(u)}{|u|} f\left(\frac{x}{u}\right) d u
$$

the boundedness of this operator taking $H^{1}(\mathbb{R})$ into $H^{1}(\mathbb{R})$ was proved in [13]; the result in [9] is a partial case of this, by letting $\varphi(x)$ to be the indicator of $[0,1]$.

Observe that in [1-3] and [16] similar problems are considered for the Hausdorff operators defined by suitable measures, while (1.1) is the partial case of absolutely continuous measures. Here we restrict ourselves to the latter case for the sake of brevity and convenience; we are aiming at a different generality, more transparent in a simpler setting. An extension to arbitrary measures goes through as in the cited papers.

Recently Hausdorff operators were considered in [7] for power series as well; in that paper the reader may find some other relevant references.

Similarly to the case of power series, the point is as follows. Since, generally speaking, the inverse formula

$$
f(x)=(2 \pi)^{-1} \int_{\mathbb{R}} \widehat{f}(t) e^{i x t} d t
$$

does not take place for $f \in L^{1}(\mathbb{R})$ as well as for $f \in H^{1}(\mathbb{R})$, where $\hat{f}$ is the Fourier transform of $f$; expected is that

$$
\int_{\mathbb{R}}(\mathcal{H} f)(y) e^{i x y} d y
$$

behaves better and characterizes $f$ properly, in a sense. For relevant relations for the Fourier transform of the value of a Hausdorff operator, see, for example, [3]. Though these operators were considered in various spaces, the most important is the case of Hardy spaces; see, for example, [13] or [15].

In the multidimensional case the situation is, as usual, more complicated. The Cesàro means in [10] and the Hausdorff means in [14] were considered in dimension 2 only for the so-called product (mixed) Hardy space $H^{11}(\mathbb{R} \times \mathbb{R}$ ) (for such spaces, see, for example, [6]), while the case of usual Hardy space $H^{1}\left(\mathbb{R}^{2}\right)$ seems to be unsolvable by the used method in full generality. In the recent paper [19] a slight extension was made in the same direction of product Hardy spaces. In [1] the problem was solved for the Hausdorff type operator

$$
\left(\mathcal{H}_{\mu} f\right)(x)=\int_{\mathbb{R}}|u|^{-n} f\left(\frac{x}{u}\right) d \mu(u),
$$

where $x \in \mathbb{R}^{n}$, defined by one-dimensional averaging. This does not seem to be natural for the multivariate case. 
The problem is to figure out an appropriate setting. We generalize (1.1) by defining a Hausdorff type operator by

$$
(\mathcal{H} f)(x)=\left(\mathcal{H}_{\Phi} f\right)(x)=\left(\mathcal{H}_{\Phi, A} f\right)(x)=\int_{\mathbb{R}^{n}} \Phi(u) f(x A(u)) d u,
$$

where $A=A(u)=\left(a_{i j}\right)_{i, j=1}^{n}=\left(a_{i j}(u)\right)_{i, j=1}^{n}$ is the $n \times n$ matrix with the entries $a_{i j}(u)$ being measurable functions of $u$. This matrix may be degenerate at most on a set of measure zero; $x A(u)$ is the row $n$-vector obtained by multiplying the row $n$-vector $x$ by the matrix $A$. This is how Hausdorff operators are defined in [3] and [16], for Borel measures rather than the Lebesgue measure. In [16] the boundedness of such operators in $H^{1}\left(\mathbb{R}^{n}\right)$ is proved for a very special case of diagonal matrices $A$ with all entries on the diagonal equal to one another.

The definition (1.2) suggests much wider range of Hausdorff type operators than those for which efficient results were obtained. We mean not only the restrictive diagonal case. For example, the above described operator from [1] (and even a variety of such operators with one-dimensional averaging) can be obtained by letting $u$ to be one-dimensional and matrix $A$ diagonal. Moreover, letting $u=\left(u_{1}, \ldots, u_{m}\right)$ with $m$ not necessarily equal to $n$, we may consider various types of averaging. We feel that the case $m=n$ is the most natural in the $n$-dimensional setting. However we do not exclude that other cases will prove to be of interest as well; our results can easily be adjusted to them.

We are going to prove sufficient conditions, in terms of $\Phi$ and $A$, for the boundedness of the whole range of Hausdorff type operators $(1.2)$ in $H^{1}\left(\mathbb{R}^{n}\right)$. This will be done in Section 2, and this is the main result of the paper.

We can easily find the adjoint operator $\mathcal{H}^{*}$ as the one satisfying, for appropriate ('good') functions $f$ and $g$,

$$
\int_{\mathbb{R}^{n}}(\mathcal{H} f)(x) g(x) d x=\int_{\mathbb{R}^{n}}\left(\mathcal{H}^{*} g\right)(x) f(x) d x .
$$

It is defined (compare [3] and [16]) as

$$
\left(\mathcal{H}^{*} f\right)(x)=\left(\mathcal{H}_{\Phi, A}^{*} f\right)(x)=\int_{\mathbb{R}^{n}} \Phi(u)\left|\operatorname{det} A^{-1}(u)\right| f\left(x A^{-1}(u)\right) d u
$$

The boundedness of this operator on $H^{1}\left(\mathbb{R}^{n}\right)$ readily follows from the main result since it is also of Hausdorff type. The key ingredient in the proofs is a lemma on the behavior in $u$ of the $B M O$-norm of $f(x A(u))$. This also allows us to get conditions for the boundedness of both operators in $B M O\left(\mathbb{R}^{n}\right)$.

In the last section we consider some examples and give concluding remarks. 


\section{Main results}

In this section we formulate and prove our main result. But before this we give natural assumptions on $\Phi$ and $A$, which provide the boundedness of the Hausdorff operator in $L^{1}\left(\mathbb{R}^{n}\right)$, that turn out to be weaker than those providing the main result. This is by no means surprising, since the latter specify only a part that belongs to $H^{1}\left(\mathbb{R}^{n}\right)$.

Let the following condition be satisfied:

$$
\|\Phi\|_{L_{A}}=\int_{\mathbb{R}^{n}}\left|\Phi(u) \| \operatorname{det} A^{-1}(u)\right| d u<\infty,
$$

or $\varphi(u)=\Phi(u) \operatorname{det} A^{-1}(u) \in L^{1}\left(\mathbb{R}^{n}\right)$.

As an example, we mention that the corresponding Cesàro operator is given by

$$
\varphi(u)=\Phi(u)\left|\operatorname{det} A^{-1}(u)\right|=\chi_{\left\{\mid \operatorname{det} A^{-1}(u) \leq \leq 1\right\}}(u) .
$$

Among the other basic properties of Hausdorff operators, one may find in [3], in different terms of course, that the operator $\mathcal{H} f$ is bounded taking $L^{1}$ into $L^{1}$, with

$$
\|\mathcal{H} f\|_{L^{1}} \leq\|\Phi\|_{L_{A}}\|f\|_{L^{1}}
$$

For diagonal matrices the belonging to $L_{A}$ either gives nothing like in [14] or coincides with the condition of belonging to $H^{1}\left(\mathbb{R}^{n}\right)$ like in [1] and [16].

There are various well-known expressions for the norm of a function in the real Hardy space $H^{1}=H^{1}\left(\mathbb{R}^{n}\right)$ (see, for example, [18, Chapters 3 and 4]). We will use the dual space approach (see, for example, [4] and [5]) in which the norm of $h \in H^{1}$ is defined as

$$
\|h\|_{H^{\prime}}=\sup _{\|g\|_{*} \leq 1}\left|\int_{\mathbb{R}^{n}} h(x) g(x) d x\right|
$$

where $g$ is taken to be infinitely smooth and of compact support and the semi-norm $\|g\|_{*}$ is that in $B M O$ :

$$
\|g\|_{*}=\sup _{Q} \inf _{c} \frac{1}{|Q|} \int_{Q}|g(x)-c| d x,
$$

where $Q$ is a cube with all the sides parallel to the coordinate axes, say admissible cube, $|Q|$ is its Lebesgue measure, and the supremum is taken over all such cubes.

We denote $\|A\|=\|A(u)\|=\max _{j}\left(\left|a_{1 j}(u)\right|+\cdots+\left|a_{n j}(u)\right|\right)$ to be the operator $\ell$-norm (see [11, Chapter IV, Section 10, Problem 244]) of the operator in an $n$-dimensional linear space defined by the matrix $A$ in the corresponding canonical basis. We will say that $\Phi \in L_{A}^{*}$ if

$$
\|\Phi\|_{L_{A}^{*}}=\int_{\mathbb{R}^{n}}|\Phi(u)|\|A(u)\|^{n} d u<\infty .
$$


LEMMA 2.1. Let $F(x, u)=f(x A(u))$. Then

$$
\|F(\cdot, u)\|_{*} \leq\|A(u)\|^{n} \mid \operatorname{det} A^{-1}(u)\|\| f \|_{*}, \quad u \in \mathbb{R}^{n} .
$$

PROOF. Changing variables, we have

$$
\begin{aligned}
\|F\|_{*} & =\sup _{Q} \inf _{c} \frac{1}{|Q|} \int_{Q}|f(x A)-c| d x \\
& =\sup _{Q} \inf _{c} \frac{\left|\operatorname{det} A^{-1}\right|}{|Q|} \int_{Q A}|f(x)-c| d x,
\end{aligned}
$$

where $Q A=Q A(u)$ is the image of $Q$ after right multiplying by $A$, the parallelepiped with the vertices that are the images of the vertices of $Q$. We now enlarge the domain of integration, $Q A$, up to the least circumscribed cube $Q_{A}$. It is easy to see that $\ell_{Q_{A}} \leq\|A\| \ell_{Q}$, where $\ell_{Q}$ denotes the side length of $Q$. Therefore,

$$
\begin{aligned}
\inf _{c} \frac{\left|\operatorname{det} A^{-1}\right|}{|Q|} \int_{Q A}|f(x)-c| d x & \leq \inf _{c} \frac{\left|\operatorname{det} A^{-1}\right|}{|Q|} \int_{Q_{A}}|f(x)-c| d x \\
& \leq \inf _{c} \frac{\|A\|^{n}\left|\operatorname{det} A^{-1}\right|}{\left|Q_{A}\right|} \int_{Q_{A}}|f(x)-c| d x \\
& \leq\|A\|^{n}\left|\operatorname{det} A^{-1}\right|\|f\|_{*},
\end{aligned}
$$

which completes the proof.

With this lemma as a tool in hand, we first obtain results on the boundedness of Hausdorff type operators in $B M O\left(\mathbb{R}^{n}\right)$.

THEOREM 2.2. (i) The Hausdorff operator $\mathcal{H} f$ is bounded on $B M O\left(\mathbb{R}^{n}\right)$ provided $\Phi \operatorname{det} A^{-1} \in L_{A}^{*}$, and $\|\mathcal{H} f\|_{*} \leq\left\|\Phi \operatorname{det} A^{-1}\right\|_{L_{A}^{*}}\|f\|_{*}$.

(ii) The adjoint Hausdorff operator $\mathcal{H}^{*} f$ is bounded on $B M O\left(\mathbb{R}^{n}\right)$ provided $\Phi \in L_{A^{-1}}^{*}$, and $\left\|\mathcal{H}^{*} f\right\|_{*} \leq\|\Phi\|_{L_{A^{-1}}^{*}}\|f\|_{*}$.

PROOF. We prove only item (i) of the theorem, the other deals with the same Hausdorff type operator with different parameters for which the changes are obvious.

Given a cube $Q$ and $\varepsilon>0$, denote by $c_{Q}(u)$ the constant satisfying

$$
\int_{Q} \mid f\left(x A(u)-c_{Q}(u)\left|d x \leq(1+\varepsilon) \inf _{c} \int_{Q}\right| f(x A(u)-c \mid d x .\right.
$$

Observe that $\left|c_{Q}(u)\right| \leq(2+\varepsilon) \int_{Q} \mid f(x A(u) \mid$, and hence

$$
c_{Q} \equiv \int_{\mathbb{R}^{n}} \Phi(u) c_{Q}(u) d u<\infty .
$$


Using (2.2), Lemma 2.1 and Fubini's theorem, we get

$$
\begin{aligned}
\frac{1}{|Q|} \int_{Q}\left|\mathcal{H} f(x)-c_{Q}\right| d x & \leq \frac{1}{|Q|} \int_{\mathbb{R}^{n}}|\Phi(u)| \int_{Q} \mid f\left(x A(u)-c_{Q}(u) \mid d x d u\right. \\
& \leq(1+\varepsilon) \int_{\mathbb{R}^{n}}|\Phi(u)|\|F(\cdot, u)\|_{*} d u \\
& \leq(1+\varepsilon)\left\|\Phi \operatorname{det} A^{-1}\right\|_{L_{A}^{*}}\|f\|_{*} .
\end{aligned}
$$

Thus, $\|\mathcal{H} f\|_{*} \leq(1+\varepsilon)\left\|\Phi \operatorname{det} A^{-1}\right\|_{L_{A}^{*}}\|f\|_{*}$. Letting $\varepsilon \rightarrow 0$ yields the desired result.

We are now in a position to obtain conditions for the boundedness of Hausdorff type operators in $H^{1}\left(\mathbb{R}^{n}\right)$.

THEOREM 2.3. (i) The Hausdorff operator $\mathcal{H} f$ is bounded on the real Hardy space $H^{1}\left(\mathbb{R}^{n}\right)$ provided $\Phi \in L_{A^{-1}}^{*}$, and $\|\mathcal{H} f\|_{H^{1}\left(\mathbb{R}^{n}\right)} \leq\|\Phi\|_{L_{A^{-1}}^{*}}\|f\|_{H^{1}\left(\mathbb{R}^{n}\right)}$.

(ii) The adjoint Hausdorff operator $\mathcal{H}^{*} f$ is bounded on the real Hardy space $H^{1}\left(\mathbb{R}^{n}\right)$ provided $\Phi \operatorname{det} A^{-1} \in L_{A}^{*}$, and $\left\|\mathcal{H}^{*} f\right\|_{H^{1}\left(\mathbb{R}^{n}\right)} \leq\left\|\Phi \operatorname{det} A^{-1}\right\|_{L_{A}^{*}}\|f\|_{H^{1}\left(\mathbb{R}^{n}\right)}$.

ProOF. As in the previous theorem, it is enough to prove only item (i). But this follows immediately from (1.3), (2.1) and from item (ii) of Theorem 2.2.

\section{Examples and concluding remarks}

In this section we collect some examples and concluding remarks.

Multidimensional Hausdorff operators investigated in previous papers were defined, in our terms, by diagonal matrices $A$. By taking such matrices with equal diagonal entries, our Theorems 2.2 and 2.3 reduce immediately to the main results from [16].

A simplest generalization to the non-diagonal case is as follows. Consider the matrix with the entries

$$
a_{i j}(u)=\frac{a_{i j}}{\psi(u)}, \quad i, j=1, \ldots, n,
$$

where $\psi(u)$ is a measurable function, $a_{i j}$ are numbers, and the corresponding matrix is non-degenerate. Setting $\Phi(u)=\varphi(u) / \psi(u)^{n}$, where $\varphi \in L^{1}\left(\mathbb{R}^{n}\right)$, we satisfy the assumptions of the theorem.

Considering, in dimension 2, the case of the diagonal matrix $A$ with $a_{j j}=1 / u_{j}$ and $a_{i j}=0, i \neq j, j=1,2$, we, correspondingly, have

$$
\Phi(u)=\frac{\varphi(u)}{\left|u_{1} u_{2}\right|},
$$


with integrable $\varphi$. By this we have the Hausdorff operator

$$
\mathcal{H}_{f}(x)=\int_{\mathbb{R}^{n}} \frac{\varphi(u)}{\left|u_{1} u_{2}\right|} f\left(\frac{x_{1}}{u_{1}}, \frac{x_{2}}{u_{2}}\right) d u
$$

considered in [14] and well adjusted to the product (mixed) Hardy space $H^{11}(\mathbb{R} \times \mathbb{R})$. Sure, we cannot expect the fulfillment of (2.1) for most of $\varphi$ in this case. The study of Hausdorff operators in general mixed Hardy spaces will appear elsewhere.

\section{References}

[1] K. F. Andersen, 'Boundedness of Hausdorff operators on $L^{p}\left(\mathbb{R}^{n}\right), H^{1}\left(\mathbb{R}^{n}\right)$, and $B M O\left(\mathbb{R}^{n}\right)^{\prime}$, Acta Sci. Math. (Szeged) 69 (2003), 409-418.

[2] G. Brown and F. Móricz, 'The Hausdorff and the quasi Hausdorff operators on the spaces $L^{p}$, $1 \leq p<\infty$, Math. Inequal. Appl. 3 (2000), 105-115.

[3] —_, 'Multivariate Hausdorff operators on the spaces $L^{p}\left(\mathcal{R}^{n}\right)^{\prime}$, J. Math. Anal. Appl. 271 (2002), 443-454.

[4] R. R. Coifman and G. Weiss, 'Extensions of Hardy spaces and their use in analysis', Bull. Amer. Math. Soc. 83 (1977), 569-645.

[5] C. Fefferman and E. M. Stein, ' $H^{p}$ spaces of several variables', Acta Math., 129 (1972), 137-193.

[6] R. Fefferman, Some recent developments in Fourier analysis and $H^{P}$ theory and product domains. II Function spaces and applications Proc. US-Swed. Semin., Lund/Swed., Lect. Notes Math. 1302 (Springer-Verlag, Berlin Heidelberg, 1988) pp. 44-51.

[7] P. Galanopoulos and A. G. Siskakis, 'Hausdorff matrices and composition operators', Ill. J. Math. 45 (2001), 757-773.

[8] C. Georgakis, 'The Hausdorff mean of a Fourier-Stieltjes transform', Proc. Amer. Math. Soc. 116 (1992), 465-471.

[9] D. V. Giang and F. Móricz, 'The Cesàro operator is bounded on the Hardy space $H^{1}$ ', Acta Sci. Math. 61 (1995), 535-544.

[10] — 'The two dimensional Cesàro operator is bounded on the multi-parameter Hardy space $\mathcal{H}^{\prime}(\mathbb{R} \times \mathbb{R})^{\prime}$, Acta Sci. Math. 63 (1997), 279-288.

[11] I. M. Glazman and Yu. I. Lyubich, Finite-Dimensional Linear Analysis: a Systematic Presentation in Problem Form (Nauka, Moscow, 1969 (Russian); English transl.: MIT Press, Cambridge, Massachusets, and London, England, 1974).

[12] G. H. Hardy, Divergent series (Clarendon Press, Oxford, 1949).

[13] E. Liflyand and F. Móricz, 'The Hausdorff operator is bounded on the real Hardy space $H^{1}(\mathbb{R})$ ', Proc. Am. Math. Soc. 128 (2000), 1391-1396.

[14] — ' The multi-parameter Hausdorff operator is bounded on the product Hardy space $H^{11}(\mathbb{R} \times$ $\mathbb{R})$ ', Analysis 21 (2001), 107-118.

[15] A. Miyachi, 'Boundedness of the Cesàro operator in Hardy spaces', J. Fourier Anal. Appl. 10 (2004), 83-92.

[16] F. Móricz, 'Multivariate Hausdorff operators on the spaces $H^{1}\left(\mathbb{R}^{n}\right)$ and $B M O\left(\mathbb{R}^{n}\right)^{\prime}$, Analysis Math. 31 (2005), 31-41.

[17] A. G. Siskakis, 'The Cesàro operator is bounded on $H^{1}$ ', Proc. Amer. Math. Soc. 110 (1990), $461-462$.

[18] E. M. Stein, Harmonic analysis: real-variable methods, orthogonality, and oscillatory integrals (Princeton Univ. Press, Princeton, NJ, 1993). 
[19] F. Weisz, 'The boundedness of the Hausdorff operator on multi-dimensional Hardy spaces', Analysis 24 (2004), 183-195.

[20] D. V. Widder, The Laplace transform (Princeton Univ. Press, Princeton, NJ, 1946).

Department of Mathematics

Bar-Ilan University

52900 Ramat-Gan

Israel

e-mail: aklerner@netvision.net.il, liflyand@macs.biu.ac.il 